\title{
National Surveillance for Acute Flaccid Myelitis — United States, 2018-2020
}

\author{
Sarah Kidd, $\mathrm{MD}^{1}$; Eileen Yee, MD ${ }^{1,2}$; Randall English, MS ${ }^{1,3}$; Shannon Rogers, MS ${ }^{1}$; Brian Emery ${ }^{1}$; Halle Getachew, MPH ${ }^{1}$; \\ Janell A. Routh, $\mathrm{MD}^{1}$; Adriana S. Lopez, MHS ${ }^{1}$
}

Acute flaccid myelitis (AFM), a recognized complication of certain viral infections, is a serious neurologic condition that predominantly affects previously healthy children and can progress rapidly, leading to respiratory insufficiency and permanent paralysis. After national AFM surveillance began in 2014, peaks in AFM cases were observed in the United States in 2014, 2016, and 2018 (1). On the basis of this biennial pattern, an increase in AFM was anticipated in 2020. To describe the epidemiology of confirmed AFM cases since 2018, demographic, clinical, and laboratory information collected as part of national AFM surveillance was reviewed. In 2018, a total of 238 confirmed AFM cases were reported to CDC, compared with 47 cases in 2019 and 32 in 2020. Enterovirus D68 (EV-D68) was detected in specimens from 37 cases reported in 2018, one case in 2019 and none in 2020. Compared with 2018, cases reported during 2019-2020 occurred in older children and were less frequently associated with upper limb involvement, febrile or respiratory prodromal illness, or cerebrospinal fluid (CSF) pleocytosis. These findings suggest that the etiologies of AFM in 2019 and 2020 differed from those in 2018. The absence of an increase in cases in 2020 reflects a deviation from the previously observed biennial pattern, and it is unclear when the next increase in AFM should be expected. Clinicians should continue to maintain vigilance and suspect AFM in any child with acute flaccid limb weakness, particularly in the setting of recent febrile or respiratory illness.

Similar to poliomyelitis caused by poliovirus (an enterovirus), AFM is characterized by sudden onset of limb weakness and lesions in the gray matter of the spinal cord. CDC began conducting national surveillance for AFM in 2014 after a cluster of cases of acute flaccid limb weakness among previously healthy children who had no laboratory or epidemiologic evidence of poliovirus infection was reported in Colorado (2). Since then, national surveillance has demonstrated biennial peaks in AFM cases during the late summer and early fall in 2014, 2016, and 2018 (1).

AFM is an unusual but known complication of certain viral infections, including those from West Nile virus and nonpolio enteroviruses $(3,4)$. Pathogens are rarely isolated from the CSF of AFM patients $(2,5)$. However, enteroviruses $(E V s)$ are the most common pathogens detected from AFM patient respiratory and stool specimens; EV-D68 is the most common enterovirus type detected, and additional laboratory and animal model data suggest that EV-D68 is the primary driver of increases in AFM during peak years (2,5-7). Previously published AFM surveillance data through 2018 suggest that case characteristics and etiology differ during peak versus nonpeak years (8). CDC examined national surveillance data to further understand the epidemiology and etiology of AFM and describe trends since 2018, the most recent peak year.

As part of national surveillance, health departments report cases meeting the clinical criterion for AFM (acute flaccid limb weakness) to $\mathrm{CDC}$ via a patient summary form that includes demographic and clinical information. Health departments also send CDC important elements from the patient's medical record, and data from these records are abstracted using a standardized worksheet. In addition, health departments and clinicians submit available CSF, respiratory, serum, and stool specimens to CDC. Testing protocols at CDC include enterovirus/rhinovirus $(\mathrm{EV} / \mathrm{RV})^{*}$ testing using methods that have been described previously (2).

Patient summary form, chart abstraction, and laboratory data were analyzed to describe trends in confirmed AFM cases since surveillance began in August 2014 and to describe case characteristics in 2018,2019, and 2020. Confirmed AFM was defined as acute onset of flaccid limb weakness accompanied by magnetic resonance imaging demonstrating a spinal cord lesion largely restricted to gray matter and spanning one or more vertebral segments (9). Reported EV/RV results include external laboratory results that were documented in the available medical records and CDC laboratory results. This activity was reviewed by $\mathrm{CDC}$ and was conducted consistent with applicable federal law and CDC policy. ${ }^{\dagger}$

A total of 238 confirmed cases were reported to CDC in 2018, 47 cases were reported in 2019, and 32 cases were reported in 2020 (Figure). During each year, at least $90 \%$ of cases (94\% in 2018 and $91 \%$ in 2019 and 2020) occurred among children aged $<18$. Compared with cases in the most recent peak year (2018), AFM patients in 2019 and 2020

\footnotetext{
${ }^{*} \mathrm{EV}$ s and RVs are closely related picornaviruses. Most available reverse transcription-polymerase chain reaction tests for EV amplify a viral gene sequence that is highly conserved between EVs and RVs. Therefore, these tests do not distinguish between EVs and RVs and additional testing is needed to identify the specific virus that has been detected.

$\dagger 45$ C.F.R. part 46.102(I)(2), 21 C.F.R. part 56; 42 U.S.C. Sect. 241(d); 5 U.S.C. Sect. 552a; 44 U.S.C. Sect. 3501 et seq.
} 


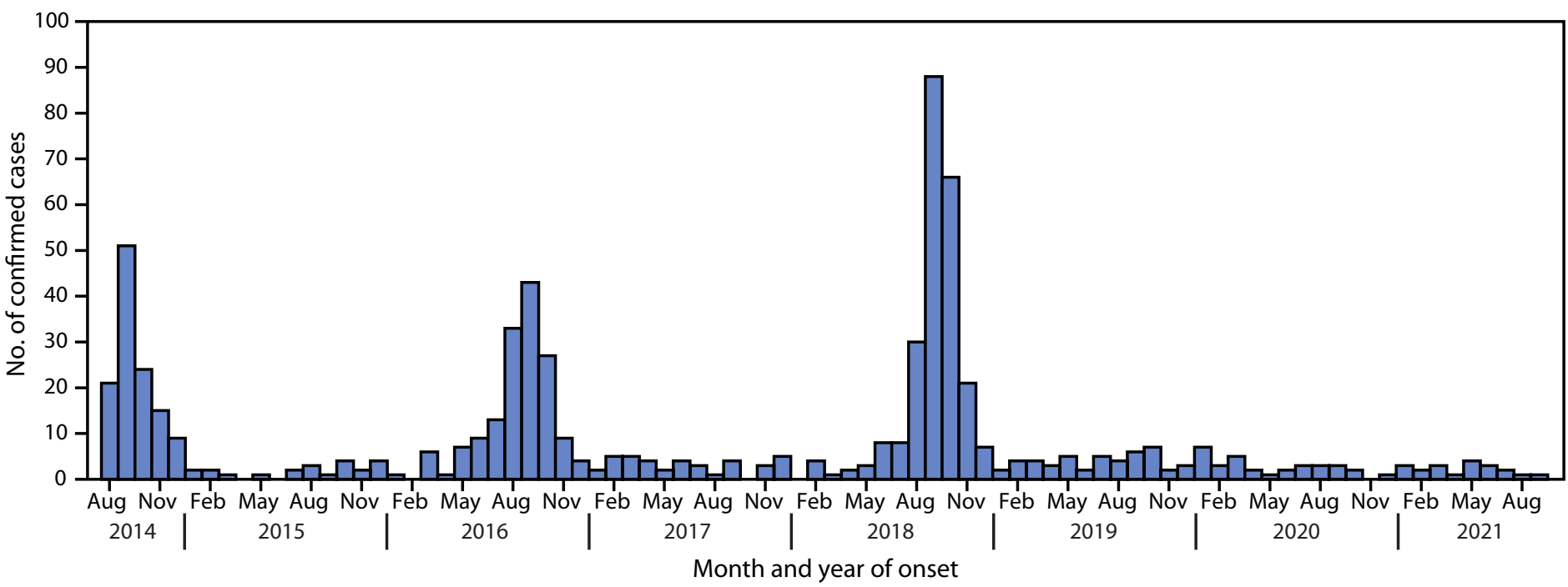

${ }^{*}$ As of October 23, 2021.

were older (median age $=6.6$ and 9.2 years, respectively, versus 5.3 years in 2018) and were less frequently associated with upper limb involvement (74\% and 59\%, versus $84 \%)$, prodromal respiratory or febrile illness $(57 \%$ and $63 \%$, versus $92 \%$ ), or CSF pleocytosis ( $49 \%$ and $48 \%$, versus $87 \%$ ) (Table 1). Lower limb involvement was more common among patients with AFM reported in 2019 and 2020 than among 2018 cases ( $74 \%$ and $81 \%$, versus $44 \%$ ).

Almost all (98\%-100\%, depending on year) patients with confirmed AFM reported during 2018-2020 were hospitalized, $51 \%-59 \%$ were admitted to an intensive care unit, and $16 \%-28 \%$ required intubation and mechanical ventilation. During each year, the emergency department was the most common location of the first medical encounter after onset of weakness. During 2018-2020, an increasing proportion of patients with confirmed AFM sought medical care and were hospitalized within 1 day of weakness onset. The percentage of patients seeking medical care within 1 day of weakness onset increased from $75 \%$ in 2018 to $97 \%$ in 2020 , and the percentage of patients hospitalized within 1 day increased from $65 \%$ to $84 \%$.

In 2018, among patients with confirmed AFM who were tested, EV/RV was detected in 50\%, and EV-D68 was the most common EV/RV type detected (Table 2). In contrast, although a similar proportion of AFM patients were tested for EV/RV each year, EV/RV was detected in only $37 \%$ of 2019 cases and in $26 \%$ of 2020 cases. EV-D68 was detected in 37 cases in 2018, compared with a single case in 2019 and no cases in 2020. During each year, the highest yield of EV/RV detection was among respiratory specimens.

\section{Summary \\ What is already known about this topic? \\ Biennial peaks in reported acute flaccid myelitis (AFM) cases occurred in late summer and early fall in 2014, 2016, and 2018. \\ What is added by this report? \\ The number of AFM cases in 2019 and in 2020 was consistent with previous nonpeak years. Compared with 2018 , cases reported during 2019-2020 were more likely to have lower limb involvement and less likely to have prodromal illness, upper limb involvement, cerebrospinal fluid pleocytosis, or detection of enterovirus D68. \\ What are the implications for public health practice? \\ It is unclear when another increase in AFM will occur. Clinicians should maintain vigilance and suspect AFM in any child with acute flaccid limb weakness, particularly following a recent febrile or respiratory illness.}

\section{Discussion}

In a departure from the previously observed pattern of biennial peaks in AFM cases in 2014, 2016, and 2018, there was no increase in the number of reported AFM cases in 2020. The number of confirmed AFM cases during 2019-2020 remained low and was consistent with previous nonpeak years. In addition, 2019-2020 cases differed from 2018 cases: patients were older; more likely to have lower limb involvement; and less likely to have upper limb involvement, prodromal illness, CSF pleocytosis, or specimens that tested positive for EV-D68. Upper limb involvement, prodromal respiratory illness, and CSF pleocytosis were characteristic features of 2018 cases. 


\begin{tabular}{|c|c|c|c|}
\hline \multirow[b]{2}{*}{ Characteristic } & \multicolumn{3}{|c|}{ No. (\%) } \\
\hline & $2018(N=238)$ & $2019(N=47)$ & $2020(N=32)$ \\
\hline Median age, yrs (IQR) & $5.3(3.3-8.2)$ & $6.6(2.9-12.8)$ & $9.2(3.5-14.5)$ \\
\hline \multicolumn{4}{|l|}{ Sex } \\
\hline Male & $138(58)$ & $15(32)$ & $16(50)$ \\
\hline Female & $100(42)$ & $32(68)$ & $16(50)$ \\
\hline \multicolumn{4}{|l|}{ Race/Ethnicity* } \\
\hline Asian & $8(3)$ & $2(4)$ & $3(9)$ \\
\hline Black or African American & $21(9)$ & $7(15)$ & $4(13)$ \\
\hline Hispanic & $47(20)$ & $12(26)$ & $8(25)$ \\
\hline Native Hawaiian or Other Pacific Islander & $1(0.4)$ & $0(-)$ & $0(-)$ \\
\hline White & $125(53)$ & $18(38)$ & $12(38)$ \\
\hline Multiracial & $4(2)$ & $1(2)$ & $0(-)$ \\
\hline Unknown & $32(13)$ & $7(15)$ & $5(16)$ \\
\hline \multicolumn{4}{|l|}{ Geographic region } \\
\hline West & $56(24)$ & $19(40)$ & $8(25)$ \\
\hline Midwest & $61(26)$ & $7(15)$ & $7(22)$ \\
\hline South & $80(34)$ & $18(38)$ & $11(34)$ \\
\hline Northeast & $41(17)$ & $3(6)$ & $6(19)$ \\
\hline \multicolumn{4}{|l|}{ Limbs affected } \\
\hline Upper & $199(84)$ & $35(74)$ & $19(59)$ \\
\hline Lower & $132(55)$ & $35(74)$ & $26(81)$ \\
\hline \multicolumn{4}{|l|}{ Illness in the 4 weeks before onset of limb weakness } \\
\hline Any illness & $223(94)$ & $32(68)$ & $20(63)$ \\
\hline Respiratory illness & $187(79)$ & $23(49)$ & $14(44)$ \\
\hline Fever & $174(73)$ & $14(30)$ & $12(38)$ \\
\hline Respiratory illness or fever & $218(92)$ & $27(57)$ & $20(63)$ \\
\hline Gastrointestinal illness & $80(34)$ & $12(26)$ & $3(9)$ \\
\hline \multicolumn{4}{|c|}{ Timing of preceding illness, median days before limb weakness ${ }^{\dagger}(\mathrm{IQR})$} \\
\hline Any illness & $5(3-8)$ & $4(3-7)$ & $5(2-13)$ \\
\hline Respiratory illness & $5(3-8)$ & $5(3-14)$ & $5.5(2.5-13.5)$ \\
\hline Fever & $3(1-5)$ & $3(2-5)$ & $2.5(1-5.5)$ \\
\hline Respiratory illness or fever & $5(3-7)$ & $4(3-6)$ & $5(2-13)$ \\
\hline Gastrointestinal illness & $2(1-6)$ & $3.5(2-6)$ & $4(0-14)$ \\
\hline \multicolumn{4}{|l|}{ CSF microscopic examination, no./total no. (\%) } \\
\hline CSF pleocytosis & $183 / 210(87)$ & $21 / 43(49)$ & $13 / 27(48)$ \\
\hline Median white blood cell count, cells $/ \mathrm{mm}^{3}(\mathrm{IQR})^{\S}$ & $94(43-163)$ & $107(44-182)$ & $36(9-55)$ \\
\hline \multicolumn{4}{|l|}{ Characteristics of hospitalization and clinical care } \\
\hline Hospitalized & $233(98)$ & $46(98)$ & $32(100)$ \\
\hline
\end{tabular}

See table footnotes on the next page.

These findings are consistent with an earlier report of differences between AFM case characteristics during peak and nonpeak years (8) and likely reflect differences in AFM etiology. Specifically, increases in AFM during peak years since 2014 appear to be mostly associated with EV-D68, whereas AFM during nonpeak years likely represents a mixture of etiologies.

Regardless of etiology, AFM can progress rapidly and lead to respiratory insufficiency that requires intubation and mechanical ventilation. Persons with signs and symptoms of AFM should be immediately hospitalized and their respiratory status monitored. Although there is no proven treatment for AFM, hospitalization facilitates patient evaluation, diagnosis or exclusion of other neurologic conditions, and appropriate medical management. Notably, during 2018-2020, the proportion of patients that were hospitalized within 1 day increased. It is possible that certain features of 2019-2020 cases (e.g., older age or lower limb predominance) facilitated earlier recognition of the signs and symptoms of neurologic weakness, although this trend might also reflect increased public and clinician awareness of AFM since 2018.

The findings in this report are subject to at least two limitations. First, this analysis was based on AFM cases reported to CDC and might underestimate the actual number of AFM cases in the United States. Second, clinical information was obtained from the patient summary form, which was completed by the health department, or from the medical records, which could be incomplete. Similarly, laboratory data were limited to results documented in the medical records shared with CDC or specimens tested at CDC. 
TABLE 1. (Continued) Demographic and clinical characteristics of patients with confirmed acute flaccid myelitis - United States, 2018-2020

No. (\%)

\begin{tabular}{|c|c|c|c|}
\hline \multirow[b]{2}{*}{ Characteristic } & \multicolumn{3}{|c|}{ No. (\%) } \\
\hline & $2018(N=238)$ & $2019(N=47)$ & $2020(N=32)$ \\
\hline \multicolumn{4}{|c|}{ Timing of hospitalization in relationship to onset of weakness among those hospitalized, no./total no. (\%) } \\
\hline Before onset of limb weakness & $25 / 233(11)$ & $6 / 46(13)$ & $1 / 32(3)$ \\
\hline After onset of limb weakness & $206 / 233(88)$ & $40 / 46(87)$ & $31 / 32(97)$ \\
\hline $\begin{array}{l}\text { Unknown if hospitalized before or after onset } \\
\text { of limb weakness }\end{array}$ & $2 / 233(1)$ & $0 / 46(-)$ & $0 / 32(-)$ \\
\hline \multicolumn{4}{|c|}{ Days from onset of weakness to hospitalization (among those hospitalized after onset of weakness), no./total no. (\%) } \\
\hline Median (IQR) & $1(0-2)$ & $1(0-1)$ & $1(1-1)$ \\
\hline $0-1$ & $134 / 206(65)$ & $33 / 40(83)$ & $26 / 31(84)$ \\
\hline $2-3$ & $52 / 206(25)$ & $5 / 40(13)$ & 4/31 (13) \\
\hline $4-7$ & $10 / 206(5)$ & $2 / 40(5)$ & $0 / 31(-)$ \\
\hline$>7$ & $10 / 206(5)$ & $0 / 40(-)$ & $1 / 31(3)$ \\
\hline \multicolumn{4}{|l|}{ Treatment } \\
\hline Steroids, no IVIG & $55(23)$ & $14(30)$ & $7(22)$ \\
\hline IVIG, no steroids & $54(23)$ & $12(26)$ & $6(19)$ \\
\hline Both steroids and IVIG & $81(34)$ & $15(32)$ & $14(44)$ \\
\hline Plasma exchange & $32(13)$ & $10(21)$ & $10(31)$ \\
\hline Admitted to ICU & $129(54)$ & $24(51)$ & $19(59)$ \\
\hline Respiratory support & $65(27)$ & $16(34)$ & $6(19)$ \\
\hline Mechanical ventilation & $55(23)$ & $13(28)$ & $5(16)$ \\
\hline \multicolumn{4}{|l|}{ Location of first medical encounter after onset of weakness } \\
\hline Emergency department & $134(56)$ & $32(68)$ & $24(75)$ \\
\hline Primary care provider & $49(21)$ & $4(9)$ & $3(9)$ \\
\hline Urgent care provider & $16(7)$ & $4(9)$ & $1(3)$ \\
\hline Had onset of weakness during an inpatient hospitalization & $25(11)$ & $6(13)$ & $1(3)$ \\
\hline Unknown or other & $14(6)$ & $1(2)$ & $3(9)$ \\
\hline \multicolumn{4}{|c|}{ Days from onset of weakness to first medical encounter (excluding those hospitalized before onset of weakness), no./total no. (\%) } \\
\hline Median (IQR) & $0(0-1)$ & $0(0-1)$ & $0(0-0)$ \\
\hline $0-1$ & $160 / 213(75)$ & $36 / 41(88)$ & $30 / 31(97)$ \\
\hline $2-3$ & $34 / 213(16)$ & $3 / 41(7)$ & $0 / 31(-)$ \\
\hline $4-7$ & $4 / 213(2)$ & 0/41 (一) & $0 / 31(-)$ \\
\hline$>7$ & $2 / 213(1)$ & $2 / 41(5)$ & $0 / 31(-)$ \\
\hline Unknown & $13 / 213(6)$ & $0 / 41(-)$ & $1 / 31(3)$ \\
\hline
\end{tabular}

Abbreviations: $\mathrm{CSF}$ = cerebrospinal fluid; ICU = intensive care unit; IQR = interquartile range; IVIG = intravenous immunoglobulin.

* Persons reported in the following groups are non-Hispanic: Asian, Black or African American, Native Hawaiian or Other Pacific Islander, and White.

† Timing calculated among cases with the prodromal illness/symptom and documented valid dates of onset.

$\S$ Median cells $/ \mathrm{mm}^{3}$ was calculated among cases with CSF pleocytosis ( $>5$ white blood cells $/ \mathrm{mm}^{3}$ ).

It is not entirely clear why AFM cases did not increase in 2020. Nonpharmaceutical interventions implemented during the COVID-19 pandemic (e.g., face masks, physical distancing, and reduced in-person school attendance) might have reduced transmission of EV-D68 and other enteroviruses associated with AFM. EV-D68 is a respiratory enterovirus, and other respiratory viruses such as influenza and respiratory syncytial virus (RSV) were noted to have decreased circulation during 2020 (10). As a group, EV/RV circulation was also attenuated, although to a lesser degree than influenza or RSV (10). It is also unclear when the next increase in AFM should be expected. AFM should be suspected in any child with acute flaccid limb weakness, especially among those with a recent history of a febrile or respiratory illness. Clinicians should remain vigilant for this condition in 2021 and report potential cases to their public health department.
Corresponding author: Sarah Kidd, skidd@cdc.gov, 404-639-8314.

${ }^{1}$ Division of Viral Diseases, National Center for Immunization and Respiratory Diseases, CDC; ${ }^{2}$ Cherokee Nation Assurance, Arlington, Virginia; ${ }^{3}$ Strategic Innovative Solutions, Clearwater, Florida.

All authors have completed and submitted the International Committee of Medical Journal Editors form for disclosure of potential conflicts of interest. No potential conflicts of interest were disclosed. 
TABLE 2. Enterovirus/rhinovirus results from respiratory, stool, cerebrospinal fluid, and serum specimens collected from patients with confirmed acute flaccid myelitis - United States, $2018^{*}-2020$

\begin{tabular}{|c|c|c|c|}
\hline \multirow[b]{2}{*}{ Specimen source } & \multicolumn{3}{|c|}{ No. (\%) } \\
\hline & $\begin{array}{c}2018 \\
(N=238)\end{array}$ & $\begin{array}{c}2019 \\
(\mathrm{~N}=47)\end{array}$ & $\begin{array}{c}2020 \\
(\mathrm{~N}=32)\end{array}$ \\
\hline \multicolumn{4}{|l|}{ Any source $^{\dagger}$} \\
\hline All patients with results & $223(94)$ & $43(91)$ & $31(97)$ \\
\hline Patients with positive results & $112(50)$ & $16(37)$ & $8(26)$ \\
\hline \multicolumn{4}{|l|}{ EV/RV type results $\S$} \\
\hline EV-D68 & 37 & 1 & 0 \\
\hline EV-A71 & 13 & 2 & 1 \\
\hline Rhinoviruses & 10 & 1 & 3 \\
\hline Other typed enteroviruses & 8 & 2 & 0 \\
\hline Unknown or not typed & 46 & 10 & 4 \\
\hline \multicolumn{4}{|l|}{ Respiratory $^{\dagger}$} \\
\hline All patients with results & $194(82)$ & $39(83)$ & $27(84)$ \\
\hline Patients with positive results & $97(50)$ & $13(33)$ & $7(26)$ \\
\hline \multicolumn{4}{|l|}{ EV/RV type results ${ }^{\S}$} \\
\hline EV-D68 & 37 & 1 & 0 \\
\hline EV-A71 & 11 & 0 & 0 \\
\hline Rhinoviruses & 10 & 1 & 3 \\
\hline Other typed enteroviruses & 1 & 0 & 0 \\
\hline Unknown or not typed & 40 & 11 & 4 \\
\hline \multicolumn{4}{|l|}{ Stool } \\
\hline All patients with results & $111(47)$ & $23(49)$ & $15(47)$ \\
\hline Patients with positive results & $25(23)$ & $6(26)$ & $2(13)$ \\
\hline \multicolumn{4}{|l|}{ EV/RV type results ${ }^{\S}$} \\
\hline EV-D68 & 3 & 0 & 0 \\
\hline EV-A71 & 2 & 2 & 1 \\
\hline Rhinoviruses & 0 & 0 & 0 \\
\hline Other typed enteroviruses & 7 & 2 & 0 \\
\hline Unknown or not typed & 13 & 2 & 1 \\
\hline \multicolumn{4}{|l|}{ Cerebrospinal fluid } \\
\hline All patients with results & $191(80)$ & $39(83)$ & $29(91)$ \\
\hline Patients with positive results & $9(5)$ & $0(-)$ & $0(-)$ \\
\hline \multicolumn{4}{|l|}{ EV/RV type results ${ }^{\S}$} \\
\hline EV-D68 & 2 & 0 & 0 \\
\hline EV-A71 & 1 & 0 & 0 \\
\hline Rhinoviruses & 0 & 0 & 0 \\
\hline Other typed enteroviruses & 0 & 0 & 0 \\
\hline Unknown or not typed & 6 & 0 & 0 \\
\hline \multicolumn{4}{|l|}{ Serum } \\
\hline All patients with results & $108(45)$ & $29(62)$ & $21(66)$ \\
\hline Patients with positive results & $4(4)$ & $0(-)$ & $1(5)$ \\
\hline \multicolumn{4}{|l|}{ EV/RV type results ${ }^{\S}$} \\
\hline EV-D68 & 1 & 0 & 0 \\
\hline EV-A71 & 0 & 0 & 1 \\
\hline Rhinoviruses & 0 & 0 & 0 \\
\hline Other typed enteroviruses & 2 & 0 & 0 \\
\hline Unknown or not typed & 1 & 0 & 0 \\
\hline
\end{tabular}

Abbreviations: $\mathrm{EV}$ = enterovirus; $\mathrm{RV}=$ rhinovirus.

* This table includes updated laboratory information and supersedes previously published data on the 2018 cases.

† Some patients had multiple positive specimens. In addition, respiratory coinfection with two EV/RV types was detected in two cases in 2018 (EV-D68 and echovirus 6 in one case, and EV-D68 and RV-A2 in another case).

$\S$ Percentage not calculated.

\section{References}

1. Lopez A, Lee A, Guo A, et al. Vital signs: surveillance for acute flaccid myelitis-United States, 2018. MMWR Morb Mortal Wkly Rep 2019;68:608-14. PMID:31295232 https://doi.org/10.15585/mmwr. mm6827e1

2. Pastula DM, Aliabadi N, Haynes AK, et al.; CDC. Acute neurologic illness of unknown etiology in children-Colorado, AugustSeptember 2014. MMWR Morb Mortal Wkly Rep 2014;63:901-2. PMID:25299607

3. Bitnun A, Yeh EA. Acute flaccid paralysis and enteroviral infections. Curr Infect Dis Rep 2018;20:34. PMID:29959591 https://doi. org/10.1007/s11908-018-0641-x

4. Solomon T, Willison H. Infectious causes of acute flaccid paralysis. Curr Opin Infect Dis 2003;16:375-81. PMID:14501988 https://doi. org/10.1097/00001432-200310000-00002

5. Sejvar JJ, Lopez AS, Cortese MM, et al. Acute flaccid myelitis in the United States, August-December 2014: results of nationwide surveillance. Clin Infect Dis 2016;63:737-45. PMID:27318332 https:// doi.org/10.1093/cid/ciw372

6. Hixon AM, Frost J, Rudy MJ, Messacar K, Clarke P, Tyler KL. Understanding enterovirus D68-induced neurologic disease: a basic science review. Viruses 2019;11:821. PMID:31487952 https://doi. org/10.3390/v11090821

7. Mishra N, Ng TFF, Marine RL, et al. Antibodies to enteroviruses in cerebrospinal fluid of patients with acute flaccid myelitis. mBio 2019;10:e01903-19. PMID:31409689 https://doi.org/10.1128/ mBio.01903-19

8. McLaren N, Lopez A, Kidd S, et al. Characteristics of patients with acute flaccid myelitis, United States, 2015-2018. Emerg Infect Dis 2020;26:212-9. PMID:31961305 https://doi.org/10.3201/eid2602.191453

9. Council of State and Territorial Epidemiologists. Revision to the standardized case definition, case classification, and public health reporting for acute flaccid myelitis. Atlanta, GA: Council of State and Territorial Epidemiologists; 2019. https://cdn.ymaws.com/www.cste. org/resource/resmgr/2019ps/final/19-ID-05_AFM_final_7.31.19.pdf

10. Olsen SJ, Winn AK, Budd AP, et al. Changes in influenza and other respiratory viral activity in the United States during the COVID-19 pandemic, 2020-2021. MMWR Morb Mortal Wkly Rep 2021;70:1013-9. PMID:34292924 https://doi.org/10.15585/mmwr.mm7029a1 